\title{
LIBERDADE PELA LEI OU LIBERDADE CONTRA A LEI EM HOBBES: FUNDAMENTOS PARA UMA TEORIA DA VONTADE
}

\author{
Delamar José Volpato Dutra \\ Universidade Federal de Santa Catarina/CNPq ${ }^{1}$
}

Resumo: 0 conceito de liberdade negativa se tornou corriqueiro na filosofia política, ao menos desde os trabalhos de Constant e Berlin. Igualmente, é quase um lugar comum referir o mesmo a Hobbes, como sendo quem por primeiro o formulou. 0 texto apresenta o referido conceito em Hobbes, a partir de duas críticas ao mesmo, quais sejam, aquela segundo a qual o seu conceito definir-se-ia em função da satisfação de desejos, de tal forma que não haveria impedimento se os desejos fossem adaptados às necessidades, e aquela segundo a qual haveria uma inconsistência na sustentação de que o medo da sanção das leis jurídicas seria um impedimento da liberdade. A resposta à primeira objeção se dará pela afirmação de que a liberdade negativamente definida como não-interferência é imune à tese do controle dos desejos, pois a vontade não é livre. Por seu turno, a resposta à segunda objeção se dará pela distinção entre a coação física da lei e o efeito oblíquo desta sobre as paixões.

Palavras-chave: Hobbes, liberdade negativa, liberdade como não-interferência, direito

Abstract: The concept of negative liberty has become a commonplace in Political Philosophy, at least since the works of Constant and Berlin. Likewise, it is almost a commonplace to refer the concept of negative liberty to Hobbes, as who first made the request. The text aims to presents the concept of negative liberty in Hobbes, considering two criticisms. The first one sustains that the concept of negative liberty should be understood as satisfaction of desires, such as that, in face of an external impediment, if it is possible to modify the desire and change it, it will be as if no impediment existed. The second critique argues for an inconsistency to support that fear of legal sanction of the laws would be an impediment to freedom, because it is not an external impediment. The answer to the first

\footnotetext{
10 autor agradece à CAPES pela bolsa "Estágio sênior no exterior" concedida no período de agosto de 2011 a julho de 2012 para o projeto de pesquisa "As críticas de Habermas a Kant e a Hobbes", realizado junto à Aberystwyth University, Wales, UK, em colaboração com Howard Williams. O autor agradece, também, à UFSC e ao seu Departamento de Filosofia pelo afastamento concedido no período mencionado.
} 
criticism is that freedom negatively defined as non-interference is immune to the objection of the control of desires, because the will is not free. The answer to the second objection sustains that there is no such appointed inconsistency if the physical coercion of the law and the fear of the coercion of the law are properly distinguished.

Keywords: Hobbes, negative liberty, liberty as non-interference, law

\section{Não-interferência}

Bentham alega ser o descobridor, em 1776, do conceito de liberdade negativa como "the absence of restraint" . Contudo, de acordo com Pettit, o conceito já houvera sido claramente formulado por Hobbes, o que mostraria que a conceituação hobbesiana ficara adormecida por mais de um século, até entrar na base das formulações liberais da liberdade. De fato, o capítulo XXI do Leviathan é o lugar clássico onde se encontra a definição hobbesiana de liberdade: "LIBERTY, or FREEDOM, signifieth (properly) the absence of Opposition; (by Opposition, I mean externall Impediments of motion;) and may be applyed no lesse to Irrationall, and Inanimate creatures, than to Rationall"'. Mais abaixo ele acrescenta que: "according to this proper, and generally received meaning of the word, A FREE-MAN, is he, that in those things, which by his strength and wit he is able to do, is not hindred to doe what he has a will to"4 . A definição hobbesiana poderia ser sumarizada na seguinte fórmula: " $\mathrm{C}$ is unfree to $\mathrm{M}$ because of constraint R"5. Desse modo, conforme o capítulo XXI, não há que se falar em liberdade da vontade, liberdade do desejo ou liberdade da inclinação, mas só da liberdade do homem, a qual consiste em que "he finds no stop, in doing what he has the will, desire, or inclination to doe". Taylor nomina de filistina essa concepção de liberdade decorrente de uma psicologia demasiadamente crua ${ }^{6}$.

\footnotetext{
2 Pettit registra escrito de Bentham nesse sentido: PETTIT, P. Republicanism: A Theory of Freedom and Government. Oxford: Oxford University Press, 1997 (1982), p. 44.

${ }^{3}$ HOBBES, T. Leviathan, or Matter, Form, and Power of a Commonwealth Ecclesiastical and Civil. (Edited by C.B. Macpherson). London: Penguin, 1968 (1651), chap. XXI.

${ }^{4}$ Idem.

${ }^{5}$ GOLDSMITH, M. M. "Hobbes on Liberty". Hobbes Studies, v. 2, n. 1, 1989, p. 25.

${ }^{6}$ TAYLOR, C. "What's Wrong with Negative Liberty". In: DYZENHAUS, D., MOREAU, S. R., RIPSTEIN, A. (Ed.). Law and Morality: Readings in Legal Philosophy. Toronto: University of Toronto Press, 2005, p. 229 e 212.
} 
Segundo Pettit, contemporaneamente, pode-se apontar para uma preponderância do conceito negativo de liberdade, como é o caso daquela que vigora na teoria da justiça de Rawls:

John Rawls manifests a concern for liberty as noninterference, for example, when he writes: 'liberty can be restricted only for the sake of liberty.' Rawls's assumption is that law always represents a restriction of liberty, and reveals a conception of liberty that is directly continuous with that of Hobbes and Bentham ${ }^{7}$.

Desse modo, o estudo do conceito de liberdade em Hobbes tem relevância sob o ponto de vista da interpretação do próprio texto de Hobbes, bem como pela recepção da mesma em outros filósofos do calibre de Rawls.

A história do conceito de liberdade negativa, em Hobbes, é marcada por paradoxos. Um deles é que a sua formulação foi cunhada para ser compatível com o absolutismo, mas acabou desempenhando papel fundamental nas teorias liberais ${ }^{8}$. Não deixa de ser irônico que o mesmo conceito de liberdade que Hobbes movimentou contra o republicanismo e a favor do absolutismo ${ }^{9}$, continha o germe de uma formulação posteriormente usada contra o próprio absolutismo ${ }^{10}$. Ou seja, no século XX alguns comentadores viram em Hobbes o gérmen do liberalismo, como é o caso de Strauss e Schmitt. Para outros comentadores, o republicanismo inglês já portava conexão com o liberalismo no sentido seguinte: "English republicans, as good liberals, wanted to make government the servant of civil society. They wanted freedom from the state, not to be incorporated in it" ${ }^{\prime 1}$.

\footnotetext{
7 PETTIT, P. Republicanism: A Theory of Freedom and Government. Oxford: Oxford University Press, 1997 (1982), p. 50.

8 PETTIT, P. Made with Words: Hobbes on Language, Mind, and Politics. Princeton and Oxford: Princeton University Press, 2008, p. 1 e 140.

9 Hobbes já foi considerado o filósofo mais importante do absolutismo: "the greatest philosopher of Absolutism" (D'ENTREVES, A. P. The Notion of the State: an Introduction to Political Theory. Oxford: Clarendon Press, 1967, p. 203).

10 PETTIT, P. Made with Words: Hobbes on Language, Mind, and Politics. Princeton and Oxford: Princeton University Press, 2008, p. 1 e 140; SKINNER, Q. "Thomas Hobbes on the Proper Signification of Liberty". Transactions of the Royal Historical Society, v. 40, 1990, p. 121-51.

11 MALIKS, R. "Prussian Polis: Kant's Democratic Republicanism". Philosophy Social Criticism, v. 35, 2009, p. 440.
} 


\section{Satisfação de desejos}

Um primeiro problema com essa conceituação negativa de liberdade decorre dos estudos de Berlin sobre a liberdade positiva e negativa. Berlin, em Four Essays on Liberty, 1969, observou que se a mencionada definição fosse compreendida como satisfação de desejos, então, uma forma de realizar tal liberdade poderia ser mediante uma espécie de autocontrole estóico dos desejos: "one of the ways of attaining such freedom is by extinguishing one's wishes. (...). If degrees of freedom were a function of the satisfaction of desires, I could increase freedom as effectively by eliminating desires as by satisfying them"12. Ou seja, tratar-se-ia da possibilidade de matar desejos, uma expressão que Berlin usa mais adiante no texto. Berlin faz essa crítica ao modo como ele formulara o conceito de liberdade negativa em Dois ensaios sobre a liberdade, 1958. É verdade que neste último texto ele referira o conceito de liberdade negativa a Hobbes ${ }^{13}$, não obstante, a liberdade como não frustração de desejos ele a imputara a Mill. Veja-se como ele se pronuncia:

In the original version of Two Concepts of Liberty I speak of liberty as the absence of obstacles to the fulfillment of a man's desires. This is a common, perhaps the most common, sense in which the term is used, but it does not represent my position. For if to be free - negatively - is simply not to be prevented by other persons from doing whatever one wishes, then one of the ways of attaining such freedom is by extinguishing one's wishes ${ }^{14}$.

Em razão dessas observações, Pettit avança o argumento de que a crítica que Berlin endereça à liberdade como não frustração de Mill deveria ser dirigida também à concepção de liberdade negativa de Hobbes ${ }^{15}$. Segundo Pettit, segue-se da definição de Hobbes que só haveria um impedimento da liberdade se a pessoa tivesse aquela vontade ou preferência específica que é negada: "being externally hindered in the choice of a given option takes from your freedom only if you have 'a will to' do it; only if

\footnotetext{
12 BERLIN, I. Liberty. Incorporating Four Essays on Liberty. (Ed. by Henry Hardy). Oxford: Oxford University Press, 2002, p. 31.

${ }^{13}$ BERLIN, I. Liberty. Incorporating Four Essays on Liberty. (Ed. by Henry Hardy). Oxford: Oxford University Press, 2002, p. 170.

14 Idem, p. 30-31.

${ }^{15}$ PETTIT, P. The Instability of Freedom as Noninterference: The Case of Isaiah Berlin. Ethics, v. 121, n. 4, 2011, p. 693-716.
} 
you prefer that option"16. Se fosse assim, poderia significar que uma autorrestrição da pessoa, por exemplo, ao modo proposto pelo estoicismo, o que é criticado por Berlin em seus textos, implicaria que ela continuaria tão livre como se não houvesse o impedimento. O importante, segundo Pettit, é que essa não seria só uma consequência implícita que Hobbes não teria percebido. Não, ele a teria sustentado explicitamente em sua discussão com Bramhall $^{17}$ : "yet it is no impediment to him that the door is shut till he have a will to play, which he has not till he has done deliberating whether he shall play or not" ${ }^{\prime 18}$. Desta última afirmação de Hobbes, Pettit conclui:

You will not be frustrated if an option you do not actually prefer — in this case, playing tennis - is blocked; you will only be frustrated if the option you prefer is obstructed. And according to Hobbes you will enjoy freedom in any choice in which you avoid such frustration ${ }^{19}$.

Pettit alega que, no texto de Berlin de 1958, intervir para obstruir uma opção não preferida seria irrelevante para a liberdade de escolha, ao passo que no texto de 1969 tal intervenção passaria a ser relevante ${ }^{20}$. Nas próprias palavras de Berlin: "For if to be free - negatively - is simply not to be prevented by other persons from doing whatever one wishes, then one of the ways of attaining such freedom is by extinguishing one's wishes" ${ }^{21}$. Em razão disso, ele defende que a liberdade não pode ser definida somente pela ausência de frustração, pois isso poderia ser conseguido pelo controle dos desejos, mas precisa também da ausência de obstáculos à possibilidade de escolher, ou seja, ela depende de quantas portas estiverem abertas para serem escolhidas:

The sense of freedom in which I use this term entails not simply the absence of frustration (which may be obtained by killing desires), but the absence of obstacles to possible choices and activities - absence of obstructions on roads along which a man

\footnotetext{
${ }^{16}$ Idem, p. 696.

17 Idem, p. 696.

18 HOBBES, T. "The Questions Concerning Liberty, Necessity, and Chance". In HOBBES, Thomas, BRAMHALL, John. Hobbes and Bramhall on Liberty and Necessity. (Ed. by Vere Chappell).Cambridge: Cambridge University Press, 1999, §25, p. 81.

19 PETTIT, P. "The Instability of Freedom as Noninterference: The Case of Isaiah Berlin". Ethics, v. 121, n. 4, 2011, p. 697.

20 Idem, p. 697-8.

${ }^{21}$ BERLIN, I. Liberty. Incorporating Four Essays on Liberty. (Ed. by Henry Hardy). Oxford: Oxford University Press, 2002, p. 31.
} 
can decide to walk. Such freedom ultimately depends not on whether I wish to walk at all, or how far, but on how many doors are open, how open they are, upon their relative importance in my life, even though it may be impossible literally to measure this in any quantitative fashion ${ }^{22}$.

Ao final, o que Pettit pretende sustentar é que a sua formulação da liberdade como não-dominação atenderia melhor ao que o próprio Berlin pretendeu com o conceito de liberdade como não-interferência, mormente na formulação que lhe dá Hobbes e que Berlin, como visto, citou explicitamente.

As perguntas que poderiam ser feitas, nesse contexto, seriam as seguintes: por que Hobbes pensou que a objeção de Bramhall à sua posição não era relevante e por que Berlin não referiu também a Hobbes a objeção feita à sua formulação da liberdade negativa do texto de 1958, mas somente a Stuart Mill?

Em primeiro lugar, porque a interpretação que Pettit imputa a Hobbes, de que só se desfruta da liberdade na qual se evita a frustração ${ }^{23}$, não procede. Isso por uma razão simples. Para Hobbes, não só não há paixões irracionais ${ }^{24}$, como não há que se falar em extinção de desejos. Falta em Hobbes, para se poder falar em extinção de desejos, o pedigree da liberdade da vontade. Destarte, do uso da expressão "livre-arbítrio" não é possível inferir qualquer liberdade da vontade, do desejo ou da inclinação, mas apenas a liberdade do homem, a qual consiste no fato de ele não se deparar com entraves ao realizar aquilo que tem vontade, desejo ou inclinação de fazer. Portanto, não se pode deduzir de is not hindred to doe what he has a will to que o sujeito possa dispor de sua vontade de fazer o que quer. Por isso, Berlin não viu a posição de Hobbes como implicando o que ele criticara na sua formulação de 1958, a saber, que a liberdade poderia ser realizada pela extinção dos desejos.

Tal ocorre pelo operar mesmo das paixões. Não há liberdade da vontade. Isso pode ser percebido no modo como Hobbes concebe a

\footnotetext{
22 Idem, p. 32.

23 "And according to Hobbes you will enjoy freedom in any choice in which you avoid such frustration" (PETTIT, P. "The Instability of Freedom as Noninterference: The Case of Isaiah Berlin". Ethics, v. 121, n. 4, 2011, p. 697).

${ }^{24}$ Hobbes afirma nunca ter usado as expressões 'sensitive appetite', 'rational hope' or 'rational fear', or 'irrational passions' (HOBBES, T. "The Questions Concerning Liberty, Necessity, and Chance". In HOBBES, T., BRAMHALL, J. Hobbes and Bramhall on Liberty and Necessity. (Ed. by Vere Chappell).Cambridge: Cambridge University Press, 1999, §25, p. 81).
} 
deliberação, a qual, como se lê no cap. VI do Leviathan, sempre ocorre conforme aos próprios apetites ou aversões. Veja-se o que ele afirma:

In Deliberation, the last Appetite, or Aversion, immediately adhæring to the action, or to the omission thereof, is that wee call the WILL; the Act, (not the faculty,) of Willing. And Beasts that have Deliberation, must necessarily also have Will. The Definition of the Will, given commonly by the Schooles, that it is a Rationall Appetite, is not good. For if it were, then could there be no Voluntary Act against Reason ${ }^{25}$.

Não há, nos termos em que o próprio Berlin critica em outras teorias, como a de Rousseau e a de Kant, um eu superior ou um eu racional que poderia se impor ou dominar um eu inferior passional. Portanto, isso proibiria completamente uma interpretação como a dos estóicos. Logo, não há que se falar em controle estóico da vontade, pois não é possível matar desejos.

Em segundo lugar, Hobbes não fala em satisfação de desejos, mas em vontade. Ora, para Hobbes, o que ocorre internamente é ditado pela causalidade das paixões, um processo necessário, como aquele operado pelo medo, o que para ele não só é compatível com a liberdade, como é a condição mesma para que a coação jurídica funcione obliquamente, ou seja, atue no cômputo das paixões para determinar o comportamento conforme ao esperado pela norma. Fosse possível matar os desejos não haveria explicação causal da vontade. $\mathrm{O}$ que ocorre com lei jurídica não é a extinção do desejo, mas, em última análise, um impedimento externo da liberdade, afinal, as paredes da prisão são um impedimento físico.

A crítica de Pettit ao conceito hobbesiano de liberdade, com base justamente nessa citação de Berlin do texto, corrigindo o que ele julgou ser um erro de formulação da liberdade negativa no texto de 1958, só seria procedente caso ele pudesse imputar a Hobbes a existência de paixões irracionais ou de um eu verdadeiro, o que é negado explicitamente por Hobbes.

\section{Da possibilidade de o medo da lei jurídica ser um impedimento}

Hobbes afirma que "a man sometimes pays his debt, only for feare of Imprisonment, which because no body hindred him from detaining, was the action of a man at liberty. And generally all actions which men doe in

${ }^{25}$ HOBBES, T. Leviathan, or Matter, Form, and Power of a Commonwealth Ecclesiastical and Civil. (Edited by C.B. Macpherson). London: Penguin, 1968 (1651), chap. VI. 
Commonwealths, for feare of the law, are actions, which the doers had liberty to omit" 26 . É o mesmo caso do homem que atira os seus bens ao mar para o barco não afundar durante uma tempestade. Seu ato é livre porque ele detinha o poder de não os jogar ao mar.

Os comentadores observam que essa formulação apontaria para uma inconsistência em Hobbes no que se refere à lei civil, pois, no caso desta, "it appears evident that it is not a body that hinders something, but a rule, norm, or law" ${ }^{27}$. De fato, isso parece ser um problema em face da formulação física dos impedimentos. Ora, Hobbes quer ofertar uma explicação causal da liberdade. Uma dificuldade com essa formulação seria como compreender os limites impostos pelos outros sobre a liberdade de alguém, um ponto importante para o conceito de liberdade como nãodominação de Pettit, bem como um ponto de suma importância para os impedimentos impostos pela lei civil. Pareceria, então, ser necessário expandir os limites dos impedimentos para além daqueles físicos para incluir os limites da lei:

But as men, for the atteyning of peace, and conservation of themselves thereby, have made an Artificiall Man, which we call a Common-wealth; so also have they made Artificiall Chains, called Civill Lawes, which they themselves, by mutuall covenants, have fastned at one end, to the lips of that Man, or Assembly, to whom they have given the Soveraigne Power; and at the other end to their own Ears. These Bonds in their own nature but weak, may neverthelesse be made to hold, by the danger, though not by the difficulty of breaking them ${ }^{28}$.

Portanto, nos termos do capítulo XXI do Leviathan, a lei é um empecilho artificial que não é difícil de desrespeitar, cujo deferência opera, portanto, pelo perigo da sanção, ou seja, o medo da pena. Porém, como afirmado há pouco, não só o súdito tem a liberdade de omitir a lei, como o medo é compatível com a liberdade. Por outro lado, Hobbes afirma no capítulo XIV do Leviathan que "Law, and Right, differ as much, as Obligation, and Liberty; which in one and the same matter are inconsistent", sem contar que no capítulo XXI há farto indício de que a lei é empecilho

\footnotetext{
${ }^{26}$ HOBBES, T. Leviathan, or Matter, Form, and Power of a Commonwealth Ecclesiastical and Civil. (Edited by C.B. Macpherson). London: Penguin, 1968 (1651), chap. XXI.

27 TRALAU, J. "Hobbes contra Liberty of Conscience". Political Theory, v. 39, n. 1, 2011, p. 69.

28 HOBBES, T. Leviathan, or Matter, Form, and Power of a Commonwealth Ecclesiastical and Civil. (Edited by C.B. Macpherson). London: Penguin, 1968 (1651), chap. XXI.
} 
para a liberdade, pois ele afirma que a liberdade está só naquilo que o soberano permite e que as liberdades dependem do silêncio da lei. Deveras, segundo Hobbes, uma possibilidade de entender a liberdade é como "aquela parte do direito de natureza que é reconhecida e deixada aos súditos pelas leis civis" 29 . Desse modo, "constitui parte substancial dessa liberdade, que é inofensiva ao governo civil, e necessária para que cada súdito viva em felicidade, que não haja penalidades a temer, a não ser as que ele possa tanto antever quanto esperar. ${ }^{" 30}$ Então, como pode a lei ser obedecida por medo da punição, o que a torna compatível com a liberdade e, ainda assim, Hobbes afirmar que ela é incompatível com a liberdade? Em consequência, parece haver uma inconsistência, pois "it is possible to be physically free to do what one is legally unfree to do"31. Skinner anota que tal expansão estaria na base de inconsistências entre uma limitação física, nos termos da qual a liberdade é definida, e a limitação pela lei, também defendida por Hobbes $^{32}$.

Como mencionado, há intérpretes que vêem uma inconsistência entre as afirmações de Hobbes de que a liberdade só é limitada por impedimentos externos e a afirmação de que a lei jurídica limita a liberdade, pois esta última seria uma limitação interna, já que ocorreria pelo medo da sanção. Para que essa inconsistência faça sentido, ela depende da afirmação de que "Laws and obligations cannot, by definition, be external impediments in the sense Hobbes originally uses the term because they cannot limit freedom in the same way that being chained to a brick wall limits freedom"33. Portanto se a pessoa não age de um certo modo por causa do medo da punição, tal não é um impedimento externo. Nesse sentido, há que se interpretar a lei como algo psicológico: "The only way that they stop a human from acting, therefore, is psychologically, either through a sense of obligation or through the fear of consequences"34. Para Mill, as leis seriam diferentes de impedimentos externos porque "they cannot stop motion in any way other than by affecting the psychological nature of the individual. For example, a

\footnotetext{
${ }^{29}$ HOBBES, T. Do cidadão. (R. J. Ribeiro: Philosophical Rudiments Concerning Government and Society). 2. ed., São Paulo: Martins Fontes, 1998 (1642), p. 107, cap. XIII, §15.

30 Idem, cap. XIII, §16.

31 GOLDSMITH, M. M. "Hobbes on Liberty". Hobbes Studies, v. 2, n. 1, 1989, p. 31.

32 SKINNER, Q. "Thomas Hobbes on the Proper Signification of Liberty". Transactions of the Royal Historical Society, v. 40, 1990, p.129.

${ }^{33}$ MILL, D. van. Liberty, rationality, and agency in Hobbes's Leviathan. Albany: State University of New York Press, 2001, p. 60.

${ }^{34}$ Idem, p. 59.
} 
law or an obligation cannot stop the motion of an animal or a falling rock" ${ }^{35}$. Dito claramente, "laws are not physical impediments"36.

Ademais, em decorrência do conceito hobbesiano de liberdade como não-interferência por impedimentos externos, Mill infere a consequência de não poder haver diferença entre o estado de natureza e o estado civil. Com efeito, "if liberty is only to be understood as the absence of external impediments, however, there is no difference in liberty in the state of nature or in society. Civil society does not put more physical impediments in our path"37.

A primeira distinção importante para tratar do assunto é aquela entre poder e liberdade. Quando não há poder para um corpo se mover, nada o impede de se mover. É o corpo que é incapaz de movimento. Um homem doente estaria incapacitado de se mover, mesmo que não estivesse preso. Isso o diferencia do prisioneiro: "He possesses an underlying power or ability to leave which has been 'taken away' from him. So while the sick man merely lacks ability, the prisoner lacks freedom, ${ }^{, 38}$. Como registra o próprio Hobbes: "But when the impediment of motion, is in the constitution of the thing it selfe, we use not to say, it wants the Liberty; but the Power to move; as when a stone lyeth still, or a man is fastned to his bed by sicknesse" ${ }^{99}$. O prisioneiro tem o poder de sair andando da prisão, mas não a liberdade para tal, pois ele está impedido pelos muros e grades, ao passo que o doente simplesmente não tem o poder para tal, logo, não há que se falar para ele em liberdade. Portanto, não havendo impedimento externo, opera o critério de poder evitar: "may refuse to doe it if he will" e "liberty to omit" ${ }^{40}$. Assim, a lei, quando opera como um impedimento interno, agiria sobre o poder de cada um, mas seria consistente com a liberdade, pois segundo a distinção por ele feita entre poder e liberdade, os impedimentos internos são compatíveis com a liberdade ${ }^{41}$, haja vista não se falar de liberdade quanto ao que ocorre internamente. Disso conclui Mill: "To

\footnotetext{
${ }^{35}$ Idem, p. 59.

${ }^{36}$ Idem, p. 61.

${ }^{37}$ MILL, D. van. Liberty, rationality, and agency in Hobbes's Leviathan. Albany: State University of New York Press, 2001, p. 60.

38 SKINNER, Q. Thomas Hobbes on the Proper Signification of Liberty. Transactions of the Royal Historical Society, v. 40, 1990, p. 127.

39 HOBBES, T. Leviathan, or Matter, Form, and Power of a Commonwealth Ecclesiastical and Civil. (Edited by C.B. Macpherson). London: Penguin, 1968 (1651), chap. XXI.

40 Idem, chap. XXI.

${ }^{41}$ MLLL, David van. Liberty, rationality, and agency in Hobbes's Leviathan. Albany: State University of New York Press, 2001, p. 56-7.
} 
maintain consistency he must mean that laws put limits upon our power, but not upon our liberty"42.

No entanto, o apelo à distinção entre poder e liberdade não se mostra hábil para resolver o problema aqui tratado, haja vista os efeitos da lei não se equipararem, nem de longe, àquilo que Hobbes entende como falta de poder. Melhor, portanto, apelar para a teoria hobbesiana da vontade, a qual tem conexão com o conceito de liberdade. Como já avançado anteriormente, o argumento é que a vontade, o desejo, as inclinações, os pensamentos, as crenças, as opiniões e a fé, não estão submetidas ao movimento, logo, por não serem passíveis de impedimento, não se lhes pode predicar a liberdade:

But when the words Free, and Liberty, are applyed to any thing but Bodies, they are abused; for that which is not subject to Motion, is not subject to Impediment: (...) Lastly, from the use of the word Free-will, no Liberty can be inferred of the will, desire, or inclination, but the Liberty of the man; which consisteth in this, that he finds no stop, in doing what he has the will, desire, or inclination to doe ${ }^{43}$.

Skinner nomina de elegante a solução de Hobbes ao problema da liberdade ${ }^{44}$, pois, ainda que a sua teoria da vontade seja marcada pela necessidade e não pela liberdade,

he is able to Mark a clear distinction between free and unfree actions. A man remains free provided that no external impediment obstructs him from acting according to his will or desire. He only ceases to be free if he is impeded in such a way that his will (which is itself caused) no longer functions as the cause of his actions ${ }^{45}$.

De fato, a vontade, o desejo, a inclinação, os pensamentos, a crença, a fé, as opiniões, não estão submetidos ao movimento, por serem despidos de corporalidade. Só o corpo humano pode se mover, portanto, só a ele se aplica o predicado da liberdade. Isso não significa que tais predicados não

\footnotetext{
42 Idem.

${ }_{43}$ HOBBES, T. Leviathan, or Matter, Form, and Power of a Commonwealth Ecclesiastical and Civil. (Edited by C.B. Macpherson). London: Penguin, 1968 (1651), chap. XXI.

${ }^{44}$ SKINNER, Quentin. Thomas Hobbes on the Proper Signification of Liberty. Transactions of the Royal Historical Society, v. 40, 1990, p. 139.

${ }^{45}$ Idem.
} 
estejam submetidos à causalidade e ao movimento interno das partículas que explicam seu funcionamento, haja vista, para Hobbes, a vida ser ela mesma nada mais do que um movimento explicável causalmente, incluso a vontade, os desejos e as inclinações, o que lhe permite conciliar o caráter necessário da vontade, do desejo e das inclinações, com a liberdade das ações. Isso ocorre quando o corpo não é impedido de agir de acordo com a sua vontade, o seu desejo e as suas inclinações, ainda que a estas determinações não se possa aplicar o predicado da liberdade ${ }^{46}$.

Sem embargo da distinção anterior, há ainda uma outra mais importante para o presente caso, a qual não foi salientada por Skinner. Segundo ele, a liberdade e a lei jurídica pertenceriam a duas esferas distintas:

liberty on the one hand, and civil law on the other, belong for Hobbes to different spheres. Liberty belongs to the sphere of nature, the sphere in which everyone has an equal right, and thus a liberty, 'to use his own power, as he will himselfe, for the preservation of his own Nature'. This liberty can only be constrained by ties or bonds which are themselves natural - that is, physical —in character. But civil law belongs to the sphere of artifice ${ }^{47}$.

Desse modo, o artifício das leis atua pelo medo, o que deixa intocada a liberdade natural: "They leave entirely unimpaired our natural liberty to make use of our powers as we please" ${ }^{, 48}$. Portanto, haveria um sentido legal e um sentido físico da liberdade, sendo este último o seu significado próprio $^{49}$.

A explicação de Skinner é procedente até o ponto em que vai. $\mathrm{Na}$ verdade, é necessário avançar para explicar em que sentido a lei jurídica se torna um empecilho para a liberdade também no sentido físico. Com efeito, a lei tem duas determinações básicas, a coação externa que funciona como um impedimento externo propriamente dito e o efeito psicológico interno sobre a paixão do medo. Segundo Beccaria, "a ignorância e a incerteza das penas propiciam a eloquência das paixões" mesmo moderado, causará sempre a impressão mais intensa que o temor de

\footnotetext{
46 Idem.

47 Idem, p. 138.

48 Idem, p. 138.

49 TRALAU, J. "Hobbes contra Liberty of Conscience". Political Theory, v. 39, n. 1, 2011, p. 69.

${ }^{50}$ BECCARIA, C.. Dos delitos e das penas. (Trad. Lucia Guidicini e Alessandro Berti Contessa: Dei delitti e delle pene). 2. ed., São Paulo: Martins Fontes, 2000 (1764), cap. 5, p. 48.
} 
outro mais severo, aliado à esperança de impunidade; pois os males, mesmo os menores, se são inevitáveis, sempre espantam o espírito humano",51. Por isso mesmo, acrescenta ele, a pena tem que ser certa e imediata para que seja relacionada ao delito. O tempo entre o delito e a pena tem que ser só aquele necessário para o processo. Aliás, essa argumentação pode ser lida no próprio Hobbes: "o medo, pelo qual se dissuade aos homens de fazer o mal, não vem do fato de haver penalidades fixadas, mas do fato de elas serem aplicadas. Pois avaliamos o futuro com base no passado, raramente esperando o que raramente acontece, ${ }^{, 52}$.

É dessa distinção que a mencionada determinação psicológica tratada por Mill é um efeito esperado da coação externa. É um efeito obliquo da certeza da coação. De tal forma que Hobbes é coerente tanto na afirmação de que a lei é compatível com a liberdade, o que ocorre quando ela opera pelo seu efeito psicológico, porque atinge a vontade da pessoa agir, mas não atinge a sua liberdade, quanto é coerente na afirmação de que ela é um impedimento da liberdade, quando ela opera como punição efetiva, normalmente depois que o preceito é descumprido, o que é uma prova, aliás, de que ela é perfeitamente compatível com a liberdade no seu modo de operar psicológico, haja vista, como o próprio Hobbes observa, em cada momento a lei poder ser desrespeitada pelo súdito.

Schmitt relembra que Feuerbach, e seu Anti-Hobbes, teria passado para a história como o autor da teoria da prevenção da coação psicológica, sujo brocado nulla poena, nullum crimen sine lege seria um resumo. Porém, observa Schmitt, "na realidade, não é mais do que um caso de aplicação dos conceitos jurídicos criados por Hobbes. (...) o 'anti-Hobbes' acaba desmascarado como Hobbes 'puro"" 53 , cuja base seria o cap. XXVII do Leviathan.

First, that where Law ceaseth, Sinne ceaseth. (...) Secondly, that the Civill Law ceasing, Crimes cease (...) Thirdly, That when the Soveraign Power ceaseth, Crime also ceaseth: for where there is no such Power, there is no protection to be had from the Law; and therefore every one may protect himself by his own

\footnotetext{
51 Idem, cap. 27, p. 92.

52 HOBBES, T. Do cidadão. (R. J. Ribeiro: Philosophical Rudiments Concerning Government and Society). 2. ed., São Paulo: Martins Fontes, 1998 (1642), p. 107, cap. XIII, §17.

${ }_{53}$ SCHMITT, Carl. El Leviathan en la teoría del estado de Tomas Hobbes. (Trad. F. J. Conde). Granada: Comares, 2004 (1938), p. 69.
} 
power: for no man in the Institution of Soveraign Power can be supposed to give away the Right of preserving his own body. ${ }^{54}$.

Segundo essa compreensão, a lei é perfeitamente um impedimento externo. Deveras, basta ver o capítulo XXVIII do Leviathan sobre a punição para se perceber que elas são todas impedimentos externos. Nesse sentido, é o próprio Locke quem afirma: "Political power, then, I take to be a right of making laws with penalties of death, and consequently all less penalties" A punição é um dano que consiste em pena corporal (como a flagelação, a pena de morte), pena pecuniária (como o confisco), ignomínia, prisão, exílio ou uma mistura destas. O próprio Mill admite que "Hobbes means by punishment the imposition of external barriers to our movements, such as prison walls and chains, and not psychological punishments, which would

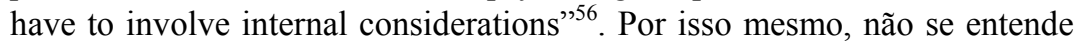
como ele pode afirmar mais adiante em seu texto que Hobbes não concebe as punições como impedimentos externos em sentido estrito: "This is not to suggest that laws are not in some sense external to the agent, but Hobbes does not count them as external impediments in the same sense as, for example, handcuffs"

Desse modo, pôr a determinação principal da lei na obrigação, como faz Mill, é dar-lhe um fundamento moral. Da mesma forma, atribuí-la ao medo sem que a coação possa se efetivar em punição externa implica um medo sem causa real, portanto, não operaria sobre a aversão por falta de causalidade. Como bem observou Beccaria, é a certeza da punição que opera sobre a paixão. A certeza da punição tem que ser necessariamente um elemento externo, físico: "Covenants, without the Sword, are but Words" Com efeito, se a lei for executada segundo sua ratio própria, pela coação ou pela espada, ela se transforma em coação física, como as penalidades

54 HOBBES, T. Leviathan, or Matter, Form, and Power of a Commonwealth Ecclesiastical and Civil. (Edited by C.B. Macpherson). London: Penguin, 1968 (1651), chap. XXVII. "Ubi lex non est, Peccatum non est.(...) Cessantibus legibus civilibus cessant crimina. (...) Ubi vero lege vel consuetudine poena limitatur, ibi majoris poenae inflictio iniqua est." (HOBBES, Thomae. Leviathan: sive de materia, forma, et potestate civitatis ecclesiasticae et civilis (1668). (Thomæ Hobbes Malmesburiensis Opera Philosophica Quæ Latine Scripsit Omnia, Volume 3, William Molesworth(ed.)). Charleston: Nabu Press, 2010, cap. XXVII, p. 211-212).

${ }^{55}$ LOCKE, J. Two Treaties of Government and A Letter Concerning Toleration. (Ed. by lan Shapiro). New Haven and London: Yale University Press, 2003 (1690 \&1689), Second Treatise, chap. I, §3.

${ }^{56} \mathrm{MILL}, \mathrm{D}$. van. Liberty, rationality, and agency in Hobbes's Leviathan. Albany: State University of New York Press, 2001, p. 57.

57 Idem, p. 59.

58 HOBBES, T. Leviathan, or Matter, Form, and Power of a Commonwealth Ecclesiastical and Civil. (Edited by C.B. Macpherson). London: Penguin, 1968 (1651), chap. XVII 
mencionadas acima. Mais que isso, se pode afirmar que o único sentido de liberdade que importa para o direito é este, qual seja, aquele da exterioridade. De fato, o direito pode obliterar o problema da liberdade da vontade e da fraqueza da vontade. Por um lado, ele trata objetivamente da responsabilidade, por exemplo, no caso brasileiro, pelo critério da idade, dezoito anos no âmbito civil, dezesseis no âmbito da cidadania e outras idades em legislação especial. Por outro lado, delega para as ciências dizerem dos casos normais, como é aquele da psiquiatria. Nem poderia ser diferente para um sistema que só pode permanecer no âmbito de uma legislação externa. Uma legislação externa pode obliterar uma tal determinação, como é o caso do sistema de Hobbes.

A vontade de um corpo resume tudo o que é interno a ele. Uma pedra não tem poder de se mover por si. Um animal tem. Isso se refere ao que se poderia chamar de potência do corpo. Assim, o fato de a limitação da lei operar internamente como medo, não significa que, no limite, a pena não poderia ser transformada em um impedimento externo. Ora, a lei jurídica é por definição externa. O que faz o medo operar de tal forma a evitar a ação ou a fazê-la, pode ser convertido em um impedimento externo, como as penas mencionadas há pouco. Não fosse assim, não haveria sentido em se falar da lei como sendo coativa.

Se no estado de natureza a lei natural vige somente em foro interno, então, a única restrição à liberdade são impedimentos externos. Pouco interessa como se determina internamente a ação. Trata-se da liberdade como um predicado do homem. No estado civil, com a vigência da lei sob a espada do poder soberano, a determinação interna da vontade continua a não ter importância fundamental. Mesmo Kant abdica dessa determinação ${ }^{59}$. A coação jurídica tem como consequência que a vontade boa não possa ser o conceito preponderante. O medo da sanção, no qual Van Mill vê tantos problemas, pode ser explicável pela prevenção que ele operará, caso o sistema seja eficiente em converter as penas em impedimentos externos da liberdade. O direito não pode impor impedimentos internos em razão da espada que o sustenta só dispor da coerção física, portanto, externa. Com isso não se quer desfazer a importância que o sistema obrigacional pode ter

\footnotetext{
59 "Se, portanto, é dito: 'Um credor tem um direito de exigir do devedor o pagamento de sua dívida', não significa isso que ele pode lhe lembrar que sua própria razão o obriga a este cumprimento, mas significa que uma coação que obriga qualquer um a fazê-lo pode muito bem coexistir com a liberdade de qualquer um, portanto também com sua própria, segundo uma lei externa universal. Direito e faculdade de coagir significam, portanto, a mesma coisa" (KANT, I. A Metafísica dos Costumes. (Tr. J. Lamego). Lisboa: Fundação Calouste Gulbenkian, 2005, p. 232)
} 
para que a espada realmente possa ter coação física, não, o que se sustenta é que tal sistema obrigacional não precisa ter base na vontade boa.

Como bem assinalou Skinner, não há inconsistência, pois a liberdade pertenceria à esfera da natureza, onde só haveria impedimentos físicos no uso do poder que cada um tem. Já a lei civil seria um artifício que não negaria esta liberdade, mas atuaria mediante a paixão do medo $0^{60}$, o que Hobbes teria resumido no próprio cap. XXI: "generally all actions which men doe in Common-wealths, for feare of the law, are actions, which the doers had liberty to omit". Ou seja, os impedimentos do direito são puramente artificiais, de tal forma que deixam "entirely unimpaired our natural liberty to make use of our powers as we please"

Portanto, a lei não é uma coação física, mas pode se transformar em uma coação física, quando, então, ela mostrará a sua natureza de impedimento no sentido estrito proposto por Hobbes, qual seja, impedimento externo. Expresso no comentário do próprio Pettit, "Obligations will be enforced by sanctions, as we know, but while sanctions may induce fear and inhibition, they will not put a block or obstruction in the way of an agent's action. Freedom of action disappears only when people are "restrained with natural impediments",62, ao que se deve emendar: se a lei jurídica não tivesse o condão de se transformar em coação física, a liberdade dos súditos não diferiria da liberdade no estado de natureza, no qual o medo também funciona, embora por outras determinações.

\section{Uma formulação jurídica da liberdade e da responsabilidade}

A interpretação fisicalista da liberdade é defensável como uma formulação jurídica da mesma no seguinte sentido. Pode-se afirmar que a finalidade mesma dessa conceituação são os impedimentos que os outros podem causar sobre a liberdade. Para isso, basta ter em vista o estado de guerra gerado no estado de natureza por efeito do uso das liberdades que cada um faz, bem como a afirmação do cap. XIV do Leviathan de que o efeito da renúncia ao próprio direito é equivalente a uma diminuição dos impedimentos para o exercício do direito natural do outro: "the effect which redoundeth to one man, by another mans defect of Right, is but so much

\footnotetext{
60 SKINNER, Q. Thomas Hobbes on the Proper Signification of Liberty. Transactions of the Royal Historical Society, v. 40, 1990, p. 138.

$61 \mathrm{ldem}$.

62 PETTIT, P. Made with Words: Hobbes on Language, Mind, and Politics. Princeton and Oxford: Princeton University Press, 2008, p. 135.
} 
diminution of impediments to the use of his own Right originall”. É cristalino, portanto, que as ações humanas podem funcionar como impedimentos, caso contrário, não haveria como explicar o grande poderio do Leviathan. Destarte, não se pode afirmar que tal definição não abarque os impedimentos decorrentes das ações humanas. "This passage also supports the argument that when Hobbes says that liberty is the absence of external impediments he seems to be thinking of impediments caused by the voluntary actions of others" $"$.

O conceito fisicalista de liberdade em Hobbes delimita um sentido mínimo de tal conceito, haja vista ele tornar, praticamente, tudo compatível com a liberdade, pois, para ele, o critério para saber se há ou não liberdade é aquele da possibilidade de agir diferentemente: "may refuse to doe it if he will", ou seja, "liberty to omit"64. Não obstante, a sua importância está justamente no que tal formulação inclui como obstáculo à liberdade, ou seja, a interferência física. Desse modo, só a coação física não passa pelo critério de compatibilidade com a liberdade. Ora, por mais estranho que soe, é justamente tal conceituação que permitirá tornar compatível a obediência à lei com a liberdade, sem que com isso seja necessário uma conceituação positiva da liberdade nos termos definidos pela lei, como sustenta o republicanismo. Se a lei é obedecida por medo ou por outro motivo interno, ela é compatível com a liberdade, pois, em razão do critério hobbesiano desta última, resta sempre a possibilidade de desobedecer a lei, logo, a lei é compatível com a liberdade, porque não retira uma possibilidade de ação. ${ }^{65}$ No limite, o sujeito poderia agir contra a lei, ainda que tivesse que suportar a pena como consequência.

Como se verá mais abaixo, isso explica por que Hobbes pôde afirmar no cap. XXI do Leviathan que o homem é igualmente livre tanto em uma monarquia quanto em um governo popular, haja vista em ambos os casos as leis poderem ser desrespeitadas, a menos que haja eficiência do sistema para exercer uma coação física sistemática, quando, então, não haveria liberdade. Não obstante, as revoltas e revoluções mostram o quanto a liberdade não deixa de ser operante em sistemas altamente repressivos. Como bem

${ }^{63}$ CURRAN, E. Reclaiming the Rights of the Hobbesian Subject. Chippenham: Palgrave Macmillan, 2007, p. 74.

64 HOBBES, T. Leviathan, or Matter, Form, and Power of a Commonwealth Ecclesiastical and Civil. (Edited by C.B. Macpherson). London: Penguin, 1968 (1651), chap. XXI.

65 "Hobbes, however, knew the difference between freedom and slavery, and the subjects of the commonwalth as He conceives them are most definitely not slaves but free human beings endowed with a number of rights." (ZAGORIN, P. Hobbes and the Law of Nature. Princeton: Princeton University Press, 2009, p. 77). 
observou Arendt ${ }^{66}$, a violência é um caso de tecnologia, de meios, ao posso que o poder é um caso de acordo. Hobbes diria, um acordo tácito daquele que não reage, mas poderia reagir.

É de se observar, também, que uma lei só tem sentido, juridicamente, se ao final ela puder impedir a liberdade em um sentido físico, como o encarceramento, o qual não só é uma pena estabelecida pela lei, mas é uma coação física que depende de muros, grilhões, e que tem o condão de impedir o ladrão de roubar e o assassino de matar, ao menos durante o tempo da prisão, o qual pode ser bem longo ou perpétuo ${ }^{67}$. Mesmo o cumprimento dos contratos manu militari implica uma coação física para aquele que é expropriado, por exemplo, via penhora. É nesse sentido mesmo que as obrigações, cujo cumprimento não possa ser executado pela coação física como um impedimento da liberdade, não passam de meras palavras. Portanto, é só nessa segunda clivagem que a lei pode ser lida como um impedimento.

É esse desdobramento da definição fisicalista da liberdade que a poucos autores chamou a atenção, mas é justamente tal formulação que terá consequências liberais posteriormente, porque ela gera um diagnóstico de que a ultima ratio da lei jurídica é uma coação física que funciona como impedimento da liberdade. O máximo que a lei pode fornecer para a liberdade é a proteção para algumas delas, contudo, quanto menos leis, mais liberdade, nos termos do próprio cap. XXI do Leviathan, haja vista a lei jurídica, por definição ter que limitar a liberdade para possibilitar a coexistência.

Harrington, logo após a publicação do Leviathan, observou em sua obra Oceana que a afirmação de Hobbes sobre a cidade de Lucca continha o equivoco de tomar a liberdade $d a$ lei pela liberdade contra a lei ${ }^{68}$ :

66 "Since violence - as distinct from power, force, or strength - always needs implements(as Engels pointed out long ago), the revolution of technology, a revolution in toolmaking, was especially marked in warfare. The very substance of violent action is ruled by the means-end category, whose chief characteristic, if applied to human affairs, has always been that the end is in danger of being overwhelmed by the means which it justifies and which are needed to reach it. Since the end of human action, as distinct from the end products of fabrication, can never be reliably predicted, the means used to achieve political goals are more often than not of greater relevance to the future world than the intended goals" (ARENDT, H. On Violence. New York, San Diego, London: Harvest/HBJ Book, Harcourt Brace Jovanovich, 1970, p. 4). "The extreme form of power is All against One, the extreme form of violence is One against All. And this latter is never possible without instruments" (Idem, p. 42).

${ }^{67}$ Para um acurado estudo da relação entre direito e coação ver: YANKAH, E. "The Force of Law: The Role of Coercion in Legal Norms". University of Richmond Law Review. v. 42, 2008, p. 1195-1255. Ver também VOLPATO DUTRA, D. J. Manual de Filosofia do Direito. Caxias do Sul: Educs, 2008.

68 "The mountain has brought forth, and we have a little equivocation! For to say that a Lucchese has no more liberty or immunity from the laws of Lucca than a Turk has from those of Constantinople; and to say 
There is written on the Turrets of the city of Luca in great characters at this day, the word LIBERTAS; yet no man can thence inferre, that a particular man has more Libertie, or Immunitie from the service of the Commonwealth there, than in Constantinople. Whether a Common-wealth be Monarchicall, or Popular, the Freedome is still the same ${ }^{69}$.

Observa-se que Hobbes usa a palavra from: "immunitie from the service". Assim, Hobbes troca a liberdade da lei, mediante a lei, que a lei concede e mantém, pela liberdade contra a lei, apesar da lei, no silêncio da lei. Essa ideia liberal do pensamento de Hobbes se tornou a base da defesa da vida privada, fundamentalmente incompatível com a sua juridicização. Talvez, um dos primeiros teóricos do direito a chamar a atenção para isso foi Bentham:

The proposition, although almost self-evident, that every law is contrary to liberty, is not generally recognized: on the contrary, the zealots of liberty, more ardent than enlightened, have made a conscience of combating it. And how have they done it? They have perverted the language, and will not employ this word in its common acceptation. They speak a language that belongs to no one: they say, Liberty consists in the power of doing every thing which does not hurt another. But is this the ordinary meaning of this word? The liberty of doing evil, is it not liberty? If it is not liberty, what is it then? and what word should we make use of in speaking of it? Do we not say that liberty should be taken away from fools, and wicked persons, because they abuse it? ${ }^{70}$

\footnotetext{
that a Lucchese has no more liberty or immunity by the laws of Lucca, than a Turk has by those of Constantinople, are pretty different speeches. The first may be said of all governments alike; the second scarce of any two; much less of these, seeing it is known that, whereas the greatest Bashaw is a tenant, as well of his head as of his estate, at the will of his lord, the meanest Lucchese that has land is a freeholder of both, and not to be controlled but by the law, and that framed by every private man to no other end(or they may thank themselves) than to protect the liberty of every private man, which by that means comes to be the liberty of the commonwealth" (HARRINGTON, J. The Commonwealth of Oceana. Constitution.org, 2006 (1656). (http://www.constitution.org/jh/oceana.htm), p. 8).

69 HOBBES, T. Leviathan, or Matter, Form, and Power of a Commonwealth Ecclesiastical and Civil. (Edited by C.B. Macpherson). London: Penguin, 1968 (1651), chap. XXI.

70 BENTHAM, J. "Principles of Morals and Legislation, Fragment on Government, Civil Code, Penal Law". In: BENTHAM, J. The Works of Jeremy Bentham (11 v.), v. 1. Edinburgh: William Tait, 1838-1843 (1780). (http://oll.libertyfund.org), p. 542. Veja-se o contraste dessa formulação de Bentham senão com Kant, pelo menos com uma interpretação possível de Kant como a de seguinte: "Autonomy replaces liberty." (HERBERT, G. B. "Kant Contra Hobbes". Hobbes Studies, v. 17, n. 1, 2004, p. 18). "Reciprocal coercion
} 
Pettit gostaria de filiar Locke à tradição segundo a qual "the right sort of law is seen as the source of liberty" "71, pois sem lei não haveria liberdade $^{72}$. Hobbes não discordaria de que a lei possibilita o exercício de algumas liberdades. $\mathrm{O}$ que se pode dizer é que não há a segurança da vida sem lei. Com isso, certamente, Hobbes concordaria, o que nos conduz à seguinte afirmação de Berlin: "Hobbes was at any rate more candid: he did not pretend that a sovereign does not enslave; he justified this slavery, but at least did not have the effrontery to call it freedom"73.

No estado de natureza, a positividade da liberdade, ou seja, o seu conceito positivo, está, nos termos do capítulo XIV do Leviathan, em "to use his own power, as he will himselfe". Trata-se do uso das suas faculdades corporais de acordo com a sua vontade. Ainda que ela seja uma definição positiva da liberdade, ela não é uma definição positiva de quando a vontade é livre. É verdade que se pode dizer que a vontade é livre quando usa o poder do corpo ao qual pertence. Não obstante, essa informação somente diz de outro modo que não há vontade sem corpo ${ }^{74}$. Para Hobbes há corpos sem vontade, mas não há vontade sem corpo. Em consequência, de acordo com Hobbes, como não se pode predicar a liberdade da vontade, mas somente a liberdade do corpo, a única definição plausível de liberdade é aquela como não-interferência com o uso que a vontade faz do corpo. Nesse sentido, a conjugação da liberdade do corpo com a não liberdade da vontade determina o conceito da liberdade como não-interferência no movimento do corpo ordenado pela vontade.

Sugere-se, por fim, a conexão do presente estudo com o pensamento de Berlin. Em Dois conceitos de liberdade, Berlin pôde concluir que a possibilidade da definição positiva da liberdade depende fundamentalmente de se considerar a vontade livre. Se só o corpo é livre, então, só se pode dizer que a liberdade é o seu movimento sem impedimento. Se a vontade é livre pode-se dizer no que consiste a sua liberdade. Por exemplo, pode-se

\footnotetext{
may restrict original (Hobbesian) liberty, but it does not infringe upon the individual's autonomy. On the contrary, reciprocal coercion constitutes recognition of the individual's autonomy." (HERBERT, G. B. Kant Contra Hobbes. Hobbes Studies, v. 17, n. 1, 2004, p. 22).

71 PETTIT, P. Republicanism: A Theory of Freedom and Government. Oxford: Oxford University Press, 1997 (1982), p. 39.

72 LOCKE, J. Two Treaties of Government and A Letter Concerning Toleration. (Ed. by lan Shapiro). New Haven and London: Yale University Press, 2003 (1690 \&1689), Second Treatise, p. 123-4, chap. VI, §57.

${ }_{73}$ BERLIN, I. Liberty. Incorporating Four Essays on Liberty. (Ed. by Henry Hardy). Oxford: Oxford University Press, 2002, p. 210.

74 FICHTE, J. G. Foundations of Natural Right According to the Principles of the Wissenschaftslehre. (Grundlage des Naturrechts nach Principien der Wissenschaftslehre: Trans.by Michael Bauer). Cambridge: Cambridge University Press, 2000 (1796-7), p. 103.
} 
dizer que a vontade é livre quando escolhe os verdadeiros interesses da pessoa (Pettit) ou quando escolhe a lei moral (Kant). Com isso, não se afirma que toda concepção segundo a qual a vontade é livre implica em definir positivamente em que consiste esta liberdade. O que se afirma é que na concepção segundo a qual a vontade não é livre não há como ofertar tal definição, por falta daquilo mesmo que deveria ser definido. Defende-se que Rousseau ${ }^{75}$ e Pettit ofertam tal definição positiva.

A formulação hobbesiana da liberdade como ausência de impedimentos externos é econômica o suficiente para evitar avanços além dos limites da experiência possível. Nesse sentido, uma proposta como a de Habermas fica a meio-caminho entre a formulação kantiana da liberdade da vontade e a negação hobbesiana da mesma. Habermas propõe tratar a causalidade mediante a liberdade nos termos de um naturalismo fraco: "along the lines of a 'weak' naturalism that integrates free will into the whole of nature" ${ }^{, 76}$. Tal naturalismo é o verso da medalha resultante de insuficiências no tratamento da relação entre a mente que opera por processos cerebrais e a influência ou determinação desses processos biológicos do cérebro sobre a mente: "The interactions between mind and brain, culture and organism remain as yet opaque",77. O que se pretende provar é que tanto uma explicação da ação baseada na liberdade da vontade, de estirpe kantiana, quanto uma baseada nas paixões, de estirpe hobbesiana, ou mesmo uma baseada em um naturalismo fraco, como a de Habermas, são explicações conteudísticas que ultrapassam os limites do dizível. Somente a definição hobbesiana da liberdade como não-interferência ou nãoimpedimento é que se mantém dentro dos limites do dizível.

Segundo Habermas, a liberdade da vontade compõe o jogo de linguagem da responsabilização das ações, pois tal jogo pressupõe a possibilidade de o ator ter agido diferentemente do que agiu: "Free will is a presupposition of the language game of responsible agency" ${ }^{\text {" }}$. A definição habermasiana de liberdade da vontade tem conexão estrita com razões vinculantes para a vontade, as quais são destiladas mediante processos argumentativos: "I understand freedom of the will, more generally, as the mode of how one binds one's own will on the basis of convincing

\footnotetext{
75 "Tous les droits sont fixés par la loi" (ROUSSEAU, J.-J. Du contrat social. Paris: Aubier, 1943 (1757), livre II, ch. VI).

${ }^{76}$ HABERMAS, J. "The Language Game of Responsible Agency and the Problem of Free Will: How can epistemic dualism be reconciled with ontological monism?". Philosophical Explorations, v. 10, n. 1, 2007, p. 17.

77 Idem.

78 Idem, p. 15.
} 
reasons" "79. Decerto, "motivation by reasons is the only form of determination that is compatible with free will" ${ }^{\prime 80}$. Ou seja, a liberdade da vontade é explicada como um modo de ser da liberdade da vontade, pois só um ato da vontade livre é capaz de se vincular à força de um argumento. A força de um argumento depende de um ato da liberdade da vontade. Ter uma convicção ou uma crença depende desse ato mesmo de uma vontade livre. Como se sabe, mesmo Hobbes nega que uma convicção ou crença possa ser imposta coativamente por meio de forças externas ao sujeito: "As for the inward thought and belief of men, which human governors take no notice of (for God only knoweth the heart), they are not voluntary, nor the effect of the laws, but of the unrevealed will and of the power of God; and consequently fall not under obligation" ${ }^{\prime \prime}$.

Assim, quando se diz que alguém poderia ter agido diferentemente se diz que este alguém poderia ter agido segundo outras razões. Da mesma forma, o significado da liberdade da vontade refere-se à conexão interna entre razões e vontade. Isso ocorre, para Habermas, quando se apela criticamente à liberdade de uma pessoa acusada de um crime para dizer que ela poderia ter agido diferentemente. Quando isso ocorre, "We charge others (or ourselves) with failing to consider the relevant reasons" ${ }^{\text {" } 2}$. Segundo ele, a liberdade da vontade teria sido reservada por Kant justamente para a conexão interna que há entre reflexão, argumentação e liberdade: "the internal connection between reflection and freedom is what explains why Kant reserves the term 'free will' or 'autonomy' for the capacity to make and carry out morally reasonable decisions" $" 83$.

Quando Habermas tenta fornecer uma explicação da liberdade da vontade percebe-se a insuficiência da linguagem para fornecer a sua explicação. Por isso, atesta ele, "The 'reflective use' of free will is an idealization" $"$. Usa-se o jogo de linguagem par excelence, qual seja, aquele

\footnotetext{
79 Idem, p. 19.

${ }^{80}$ Idem, p. 20.

81 HOBBES, T. Leviathan, or Matter, Form, and Power of a Commonwealth Ecclesiastical and Civil. (Edited by C.B. Macpherson). London: Penguin, 1968 (1651), chap. XL. "But what(may some object) if a King, or a Senate, or other Soveraign Person forbid us to beleeve in Christ? To this I answer, that such forbidding is of no effect; because Beleef, and Unbeleef never follow mens Commands" (HOBBES, T. Leviathan, or Matter, Form, and Power of a Commonwealth Ecclesiastical and Civil. (Edited by C.B. Macpherson). London: Penguin, 1968 (1651), chap. XLII).

82 HABERMAS, Jürgen. The Language Game of Responsible Agency and the Problem of Free Will: How can epistemic dualism be reconciled with ontological monism? Philosophical Explorations, v. 10, n. 1, 2007, p. 19.

${ }^{83}$ Idem, p. 18.

${ }^{84}$ Idem, p. 17.
} 
do desempenho discursivo de uma pretensão de validade, sobre o qual é possível falar reflexivamente ou reconstrutivamente, para falar de algo que transcende o uso possível da linguagem. Ou seja, trata-se de um modo oblíquo, indireto, de ofertar uma explicação de como razões operariam no sujeito: "The freedom to say 'yes' or 'no' to validity claims, and especially the freedom involved in processes of rational will-formation, can be analyzed as the capacity to make commitments exclusively on the basis of insight" ${ }^{85}$. Mais que isso, Habermas conjuga o conceito de vontade boa e de insight, o que faz a liberdade que opera na racionalidade comunicativa depender de uma característica moral da vontade do agente: "Good intentions motivated by practical insights need to be translated into actions, if we are to speak of "causality through freedom"" ${ }^{\prime \prime 6}$. Ao final, tem-se um sentido intuitivo, obliquo do que seja liberdade da vontade: "The intuitive sense of having a free will mirrors a pragmatic presupposition of language games" ${ }^{\prime 87}$, o qual, em geral, opera retrospectivamente, ou seja, quando se passa a analisar as ações feitas no passado e quais foram os seus motivos. Isso ocorre porque, na maior parte dos casos, as ações são irrefletidas. Simplesmente se age. Por isso mesmo, observa ele, pode haver responsabilização por ações não refletidas que decorrem do caráter de alguém, bem como por aquelas que ocorrem por negligência. Em tais casos, a culpa é geral. Ela não equivale ao dolo. Ou seja, se a pessoa tivesse refletido, provavelmente ela agiria diferentemente. Contudo, ela é responsabilizada pela culpa geral de não ter sido diligente o suficiente.

A explicação de Hobbes também pode ser considerada uma explicação oblíqua de processos interiores, explicação esta que exorbita da sua definição de liberdade negativa como não-interferência. Deveras, em relação a um exemplo do próprio Hobbes, poder-se-ia perguntar, o que ocorre internamente com aquele que decide morrer a matar o próprio filho? Pode ser que a aversão de matar o filho seja maior do que a aversão de morrer. Em qualquer caso, trata-se da predominância da maior aversão, o que se configura caso a caso, assim como no movimento de um rio, a depender de alguma pedra no seu leito ou reentrância nas margens, pode fazer que parte da água volte e, depois, continue a descer. Uma pedra quando rola, pode pular se bater em outra, e mesmo voltar para trás neste mesmo caso. Em todas essas situações, trata-se sempre de movimento e de causalidade. Da mesma forma em relação à vontade.

\footnotetext{
${ }^{85}$ Idem, p. 17.

86 Idem p. 17.

${ }^{87}$ Idem, p. 18.
} 
Para um jurista, a paixão, por mais violenta que seja, não exclui a imputabilidade. Hobbes pode ser lido, em relação à negação da liberdade da vontade, como uma concepção deflacionada de vontade, sem ranços metafísicos. Ou seja, para o direito não faz diferença se há ou não vontade boa, se há ou não liberdade da vontade. Não precisa uma profundidade da consciência desse jaez para estabelecer a responsabilidade. Basta a experiência de que certos corpos reagem de um certo modo a normas com ameaça de sanção. Uma tal ameaça não funciona para um cavalo, como não funciona para certos seres humanos, como os bebês ou os loucos. Para tal, não há outro caminho senão a experiência para estabelecer os casos, via psiquiatria. Mesmo o que admite a liberdade da vontade ou estabelece um pressuposto metafísico para além da experiência possível tem que apelar à ciência psiquiátrica, tanto quanto aquele que nega a liberdade da vontade. Os conceitos de liberdade e de vontade em Hobbes são suficientemente deflacionados para que sejam tratados a partir do conceito de normalidade, entendida de forma relacional e social. Deveras, eis o que ele afirma no que se refere à loucura: "and to have stronger, and more vehement Passions for any thing, than is ordinarily seen in others, is that which men call MADNESSE" ${ }^{\prime 88}$. Como se pode ver, o conceito de loucura é relacional, ou seja, deve ser averiguado em relação ao que se considera ocorrer na maior parte dos casos em relação ao corpo dos outros. Da mesma forma em relação à autoria das ações:

A PERSON, is he, whose words or actions are considered, either as his own, or as representing the words or actions of an other man, or of any other thing to whom they are attributed, whether Truly or by Fiction. When they are considered as his owne, then is he called a Naturall Person: And when they are considered as representing the words and actions of an other, then is he a Feigned or Artificiall person. ${ }^{89}$

Veja-se que o termo que ele usa é "considered". Ou seja, trata-se de algo socialmente estabelecido. Hobbes não precisa se comprometer com teses metafísicas a respeito da liberdade da vontade.

Nesse diapasão, veja-se o que Hart sustenta sobre o modo como a responsabilidade é tratada juridicamente por comparação ao modo como ela é

\footnotetext{
88 HOBBES, T. Leviathan, or Matter, Form, and Power of a Commonwealth Ecclesiastical and Civil. (Edited by C.B. Macpherson). London: Penguin, 1968 (1651), chap. VIII.

${ }^{89}$ Idem, chap. XVI.
} 
tratada moralmente. De acordo com ele, a responsabilidade objetiva, ou se-ja, sem consideração da liberdade da vontade, não é um anatocismo jurídico:

If someone does something forbidden by moral rules or fails to do what they require, the fact that he did so unintentionally and in spite of every care is an excuse from moral blame; whereas a legal system or custom may have rules of 'strict liability' under which those who have broken the rules unintentionally and without 'fault' may be liable to punishment ${ }^{90}$.

Da mesma forma, o pressuposto da mens rea ${ }^{91}$ é de fundamental importância para a responsabilização moral ${ }^{92}$, não para a jurídica, pois esta última, frente às dificuldades de provar fatos psicológicos, torna a responsabilidade independente do pressuposto da mens rea: "Again, the legal system may, for certain types of offence, impose 'strict liability' and make responsibility independent of mens rea altogether, except perhaps for the minimum requirement that the accused must possess normal muscular control" ${ }^{\prime 23}$.

A formulação hobbesiana da liberdade é econômica porque ela permite inferir do fato de não haver impedimento externo a uma conduta a conclusão de que esta é uma razão bastante para sustentar que o agente poderia ter agido diferentemente. É econômica porque tal inferência pode ser feita sem que seja necessário tratar do que ocorre internamente, seja por força ${ }^{94}$ da liberdade da vontade, seja por força das paixões. Por isso, sugere-se distinguir (i) a definição negativa de liberdade, a qual é, certamente, imune a teses metafisicas, e (ii) a explicação naturalizada de Hobbes de como opera a vontade via paixões. Assim como Hobbes apela para as paixões, a análise de Habermas do modo como

\footnotetext{
90 HART, H. L. A. The Concept of Law. 2. ed., Oxford: Clarendon Press, 1994 (1961), p.173.

91 "Actus non facit reum nisi mens sit rea", o que significa: "o ato não torna a pessoa culpável a menos que a mente também seja culpável".

92 "Moral blame is therefore excluded because he has done all that he could do. In any developed legal system the same is true up to a point; for the general requirement of mens rea is an element in criminal responsibility designed to secure that those who offend without carelessness, unwittingly, or in conditions in which they lacked the bodily or mental capacity to conform to the law, should be excused. A legal system would be open to serious moral condemnation if this were not so, at any rate in cases of serious crimes carrying severe punishments" (HART, H. L. A. The Concept of Law. 2. ed., Oxford: Clarendon Press, 1994 (1961), p. 178).

${ }^{93}$ HART, H. L. A. The Concept of Law. 2. ed., Oxford: Clarendon Press, 1994 (1961), p. 178.

94 "judgment. Self-determination means having the strength of will to ensure that, in acting, one is determined by precisely those reasons that one has found convincing oneself" [HABERMAS, J. "The Language Game of Responsible Agency and the Problem of Free Will: How can epistemic dualism be reconciled with ontological monism?". Philosophical Explorations, v. 10, n. 1, 2007, p. 16).
} 
opera a liberdade pressuposta pela racionalidade comunicativa apela a insights. Sob o ponto de vista da imputação, não faz diferença.

Mas, então, por que um animal ou uma pedra não são imputáveis? A imputação em Hobbes tem a ver com a normalidade daquele que pode calcular as consequências da sanção, por exemplo, por medo dela. Disso se segue que Hobbes poderia concordar com a tese de que seria possível haver responsabilização penal mesmo sem a liberdade da vontade, como um cálculo para a proteção da ordem pública. Porém, ainda que dê a mão à palmatória a Hobbes, Habermas não pode concordar com a tese básica daquele: sem a liberdade da vontade "prosecution no longer makes any sense-except for the purpose of protecting society"

\section{Referências}

ARENDT, H. On Violence. New York, San Diego, London: Harvest/HBJ Book, Harcourt Brace Jovanovich, 1970.

BECCARIA, C. Dei delitti e delle pene (Letteratura italiana Einaudi). Mursia: Renato Fabietti, 1973 (1764).

. Dos delitos e das penas (Trad. Lucia Guidicini e Alessandro Berti Contessa: Dei delitti e delle pene). 2.ed. São Paulo: Martins Fontes, 2000 (1764).

BENJAMIN CONSTANT,. The Liberty of Ancients Compared with that of Modern (1816). Indianapolis: Liberty Fund.

(http://files.libertyfund.org/files/2251/Constant_Liberty1521_EBk_v6.0.pdf ) .

BERLIN, I. Freedom and its Betrayal: Six Enemies of Human Liberty (Edited by Henry Hardy). London: Chattus \& Windus, 2002 (1952).

. Liberty. Incorporating Four Essays on Liberty (Ed. by Henry Hardy). Oxford: Oxford University Press, 2002.

BRASIL. Decreto-Lei n. 2.848, de 7 de dezembro de 1940. Código Penal.

D'ENTREVES, A. P. The Notion of the State: an Introduction to Political Theory. Oxford: Clarendon Press, 1967.

FICHTE, J. G. Foundations of Natural Right According to the Principles of the Wissenschaftslehre. (Grundlage des Naturrechts nach Principien der Wissenschaftslehre: Trans. by Michael Bauer). Cambridge: Cambridge University Press, 2000 (1796-7).

\footnotetext{
${ }^{95}$ HABERMAS, J. "The Language Game of Responsible Agency and the Problem of Free Will: How can epistemic dualism be reconciled with ontological monism?". Philosophical Explorations, v. 10, n. 1, 2007, p. 20.
} 
GAUTHIER, D. The Logic of Leviathan. Oxford: Clarendon Press, 1969. GERT, B. "Hobbes on Reason". Pacific Philosophical Quarterly, v. 82, 2001, p. 243-57.

GOLDSMITH, M. M. "Hobbes on Liberty". Hobbes Studies, v. 2, n. 1, 1989, p. 23-39.

HABERMAS, J. "The Language Game of Responsible Agency and the Problem of Free Will: How can epistemic dualism be reconciled with ontological monism?". Philosophical Explorations, v. 10, n. 1, 2007, p. 1350.

HARRINGTON, J. The Commonwealth of Oceana. Constitution.org, 2006 (1656). (http://www.constitution.org/jh/oceana.htm).

HOBBES, T. Leviathan: sive de materia, forma, et potestate civitatis ecclesiasticae et civilis (Thomæ Hobbes Malmesburiensis Opera Philosophica Quæ Latine Scripsit Omnia, Volume 3, William Molesworth (ed.)). Charleston: Nabu Press, 2010 (1668).

HOBBES, T. Leviathan, or Matter, Form, and Power of a Commonwealth Ecclesiastical and Civil (Edited by C.B. Macpherson). London: Penguin, 1968 (1651).

. "The Questions Concerning Liberty, Necessity, and Chance". In: . BRAMHALL, John. Hobbes and Bramhall on Liberty and Necessity. (Ed. by Vere Chappell). Cambridge: Cambridge University Press, 1999, p. 69-90.

KAPUST, D. J.; TURNER, B. P. "Democratical Gentlemen and the Lust for Mastery: Status, Ambition, and the Language of Liberty in Hobbes's Political Thought". Political Theory. Vol. XX, published online 15 May 2013, p. 1-28.

LOCKE, J. Two Treaties of Government and A Letter Concerning Toleration. (Ed. by Ian Shapiro). New Haven and London: Yale University Press, 2003 (1690 \& 1689).

MILL, D. van. Liberty, rationality, and agency in Hobbes's Leviathan. Albany: State University of New York Press, 2001.

PETTIT, P. "Free Persons and Free Choices". History of Political Thought, v. 28, 2007, p. 709-718.

. "Freedom and Probability: A Comment on Goodin and Jackson". Philosophy and Public Affairs. n. 36, 2008, p. 206-20.

"Keeping Republican Freedom Simple". Political Theory, v. 30, 2002, p. 339-56.

. Made with Words: Hobbes on Language, Mind, and Politics. Princeton and Oxford: Princeton University Press, 2008. 
. "Neo-Republicanism: A Normative and Institutional Research Program". In: Annual Review of Political Science, v. 12, 2009, p. 11-29. . Republicanism: A Theory of Freedom and Government. Oxford: Oxford University Press, 1997 (1982).

. "The Instability of Freedom as Noninterference: The Case of Isaiah Berlin". Ethics, v. 121, n. 4, 2011, p. 693-716.

SKINNER, Q. "The Ideological Context of Hobbes's Political Thought". In: The Historical Journal, v. 9, n. 3, 1966, p. 286-317.

"Thomas Hobbes on the Proper Signification of Liberty". Transactions of the Royal Historical Society, v. 40, 1990, p. 121-51.

TAYLOR, C. "What's Wrong with Negative Liberty". In: DYZENHAUS, D., MOREAU, S. R., RIPSTEIN, A. (Ed.). Law and Morality: Readings in Legal Philosophy. Toronto: University of Toronto Press, 2005, p. 359-368.

TRALAU, Johan. "Hobbes contra Liberty of Conscience". Political Theory, v. 39 , n. 1,2011 , p. $58-84$.

VOLPATO DUTRA, D. J. Manual de Filosofia do Direito. Caxias do Sul: Educs, 2008.

WARRENDER, H. The Political Philosophy of Hobbes. Oxford: Oxford University Press, 1957.

WENDT, F. "Slaves, Prisoners, and Republican Freedom". Res Publica, v. 17, 2011, p. 175-192.

YANKAH, E. N. "The Force of Law: The Role of Coercion in Legal Norms". University of Richmond Law Review. v. 42, 2008, p. 1195-1255. ZAGORIN, P. Hobbes and the Law of Nature. Princeton: Princeton University Press, 2009. 